\title{
COCCOPHAGINAE (HYMENOPTERA: CHALCIDOIDEA: APHELINIDAE) DE MÉXICO CON CLAVE DE GÉNEROS Y DESCRIPCIÓN DE UNA NUEVA ESPECIE
}

\section{Svetlana N. MYARTSEVA, ENRique RUIZ-CANCINO* y JuANa María CORONADO-BLANCO}

\author{
Facultad de Ingeniería y Ciencias, Universidad Autónoma de Tamaulipas, Centro Universitario Adolfo López \\ Mateos, 87149 Ciudad Victoria, Tamaulipas, México, *Autor de correspondencia: <eruiz@uat.edu.mx>
}

Recibido: 26/01/2015; aceptado: 08/12/2015

\begin{abstract}
Myartseva, S.N., Ruiz-Cancino, E. \& Coronado-Blanco, J.M. 2016. Coccophaginae (Hymenoptera: Chalcidoidea: Aphelinidae) de México con clave de géneros y descripción de una nueva especie. Acta Zoológica Mexicana (n. s.), 32(1): 6-11.
\end{abstract}

RESUMEN. La subfamilia Coccophaginae en México contiene tres tribus y seis géneros, contribuye con alrededor del $70 \%$ de la fauna mexicana de la familia Aphelinidae. En esta subfamilia se encuentran los géneros más diversos, Encarsia Förster (108 especies) y Coccophagus Westwood (30 especies). Se elaboró una clave para los géneros presentes en México. Se describió una nueva especie, Coccobius frontalis sp. nov.

Palabras clave: Aphelinidae, Coccophaginae, tribus, géneros, clave, nueva especie.

\section{INTRODUCCIÓN}

La familia Aphelinidae es una de las familias de Chalcidoidea (Hymenoptera) que incluye actualmente 1345 especies y 39 géneros en las principales regiones zoogeográficas -Neotropical, Holártica, Etiópica, Oriental, Oceánica y Australiana (Noyes 2014, Myartseva et al. 2014). En México se conocen 205 especies y 13 géneros de cuatro subfamilias -Aphelininae, Calesinae, Azotinae y Coccophaginae (Myartseva et al. 2012). De las 6 subfamilias de Aphelinidae, Coccophaginae con 4 tribus y 13 géneros, es una de las más diversas en especies (Hayat, 1998). A nivel mundial, esta subfamilia incluye el $65.8 \%$ de la fauna de Aphelinidae y alrededor del 70\% en México. Las especies de Coccophaginae son parasitoides de Hemiptera, predominantemente de las familias Coccidae, Diaspididae y Aleyrodidae; en México, las especies de la tribu Coccophagini atacan Coccidae, las de la tribu Pteroptricini parasitan Aleyrodidae y Diaspididae mientras que las de la tribu Physcini son enemigos naturales de Coccidae y Diaspididae (Myartseva et al. 2012). El género Coccobius Ratzeburg es conocido en México solamente desde hace 15 años (Myartseva, 2000). Por su parte,
Myartseva, S.N., Ruiz-Cancino, E. \& Coronado-Blanco, J.M. 2016. Mexican Coccophaginae (Hymenoptera: Chalcidoidea: Aphelinidae) with a key to genera and description of a new species. Acta Zoológica Mexicana (n. s.), 32(1): 6-11.

ABSTRACT. The subfamily Coccophaginae in Mexico consists in three tribes and six genera, and comprises about $70 \%$ of Mexican fauna of the family Aphelinidae. This subfamily includes the most diverse genera, Encarsia Förster and Coccophagus Westwood with 108 and 30 Mexican species, respectively. A key to the genera of Coccophaginae in Mexico is provided. A new species, Coccobius frontalis sp. nov., is described and illustrated.

Key words: Aphelinidae, Coccophaginae, tribes, genera, key, new species.

el género Mexidalgus Myartseva fue descrito en 2014 (Myartseva et al. 2014).

Los objetivos de este artículo fueron aportar información sobre la subfamilia Coccophaginae y su distribución en México, elaborar una clave de géneros y describir una nueva especie de Coccobius.

\section{MATERIALES Y MÉTODOS}

Se recibieron especímenes de Coccobius en préstamo del Entomological Research Museum de la Universidad de California-Riverside (UCRC Ent.) para su estudio e identificación. Los insectos habían sido colectados en 'pan traps' en el Estado de Baja California Sur, México, en abril-junio de 1984-1986 en Barracas, localidad situada en el extremo sur de la Península de Baja California. El especimen de la nueva especie estaba montado en seco. En el Museo de Insectos de la Facultad de Ingeniería y Ciencias en la Universidad Autónoma de Tamaulipas (UAT), Cd. Victoria, Tamaulipas, México, fue montado de nuevo en laminilla con bálsamo de Canadá para estudiar la morfología e identificación de este espécimen 
extremadamente pequeño. Se utilizó un microscopio compuesto Leica GME con aumentos de 280x y 400x. Para la identificación se usaron las claves de Hayat (1984, 1998), Hayat \& Khan (2010), Prinsloo (1995), Wang et al. (2013), Myartseva (2000) y las descripciones originales de especies similares. Los materiales estudiados de las especies de seis géneros de Coccophaginae están preservados en el Museo UAT.

\section{RESULTADOS Y DISCUSIÓN}

De los 13 géneros y 4 tribus de Coccophaginae que existen (Hayat 1998), en México se han encontrado 6 géneros y 3 tribus (la tribu Euxanthellini no se ha colectado), incluyendo el género Mexidalgus descrito en 2014 (Myartseva et al. 2012, 2014). Los géneros Encarsia (108 especies) y Coccophagus (30 especies) son los más diversos, estando más ampliamente distribuídos en México que los otros géneros (Cuadro 1).

\section{Clave de géneros de Coccophaginae de México}

1. Todos los tarsos de 4 segmentos. ... Pteroptrix Westwood

- Todos los tarsos de 5 segmentos o mesotarso de 4 seg-

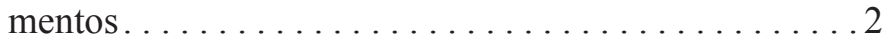

2. Flagelo antenal de la hembra con 5 segmentos, flagelo antenal del macho con 6 segmentos; cerci con 3 setas largas de casi el mismo tamaño...... Coccobius Ratzeburg - Flagelo antenal de la hembra con 6 segmentos (5 en Mexidalgus), antena del macho con 5 o 6 segmentos; cerci con 2 setas largas y una seta relativamente corta . . . . 3 3. Escutelo con 4 setas, axilas con 1 seta .........4 - Escutelo con 6 o más setas, axilas con 2 o más setas . 5
4. Mesoescudo con lóbulos laterales divididos. . Dirphys Howard

- Mesoescudo con lóbulos laterales no divididos.................... Encarsia Förster 5. Flagelo antenal de la hembra con 5 segmentos; mandíbula aserrada en el margen apical.Mexidalgus Myartseva - Flagelo antenal de la hembra con 6 segmentos; mandíbula no aserrada en el margen apical .... Coccophagus Westwood

\section{Géneros de Coccophaginae en México Tribu Coccophagini Förster}

Diagnosis. Pronoto entero. Axilas grandes, subtriangulares, proyectadas hacia adelante; distancia interaxilar no mayor que la longitud de una axila; cada axila con al menos 2 setas. Escutelo grande, no más de 1.5 veces más ancho que largo y con al menos 6 setas. Vena submarginal con 5 o más setas. Generalmente son parasitoides de escamas suaves. En México, esta tribu incluye solamente al género Coccophagus.

\section{Género Coccophagus Westwood}

Coccophagus Westwood, 1833b: 344.

Aneristus Howard, 1895b: 351.

Paracharitopus Brèthes, 1913: 105.

Ataneostigma Girault, 1914: 23.

Prococcophagus Silvestri, 1915a: 359

Taneostigmoidella Girault, 1915: 39.

Euxanthellus Silvestri, 1915a: 320.

Onophilus Brèthes, 1918: 155.

Parencarsia Mercet, 1930a: 198.

Heptacritus De Santis, 1960: 97.

Aclerdaephagus Sugonjaev, 1969: 290.

Polycoccophagus Sugonjaev, 1976: 106.

Dicoccophagus Sugonjaev, 1994: 427-428.

Cuadro 1. Distribución y hospederos de los géneros de Coccophaginae en las entidades federativas mexicanas.

\begin{tabular}{lll}
\hline Géneros & Hospederos & Distribución \\
\hline $\begin{array}{l}\text { Coccobius } \text { Ratzeburg } \\
\text { Coccophagus Westwood }\end{array}$ & Diaspididae & Baja California Sur, Nuevo León, San Luis Potosí, Tamaulipas. \\
& & Aguascalientes, Baja California Sur, Campeche, Chiapas, Chihuahua, Distrito \\
& & Federal, Guanajuato, Guerrero, Jalisco, Michoacán, Morelos, Nuevo León, \\
Dirphys Howard & Aleyrodidae & Tabasco, San Luis Potosí, Sinaloa, Tamaulipas, Veracruz. \\
Encarsia Förster & Aleyrodidae, Diaspididae & Baja California Sur, Chiapas, Chihuahua, Coahuila, Colima, Distrito Federal, \\
& & Guanajuato, Guerrero, Jalisco, Michoacán, Morelos, Nayarit, Nuevo León, \\
& & Oaxaca, Puebla, Querétaro, Quintana Roo, San Luis Potosí, Sinaloa, Tabasco, \\
& Tamaulipas, Veracruz, Yucatán. \\
Mexidalgus Myartseva & Coccidae & Hidalgo, Veracruz. \\
Pteroptrix Westwood & Diaspididae & Baja California Sur, Chiapas, Michoacán, Morelos. \\
\hline
\end{tabular}


La fauna mundial de este género incluye 259 especies descritas (Noyes 2014). Sus especies son endoparasitoides of Coccoidea, principalmente de escamas suaves (Coccidae) y raramente de piojos harinosos (Pseudococcidae). Generalmente, los machos son hiperparasitoides de otros parasitoides primarios, incluyendo a las hembras de su propia especie. En México se conocen 30 especies (Myartseva et al. 2014).

\section{Tribu Pteroptricini Ashmead}

Diagnosis. Pronoto membranoso medialmente. Axilas elongadas, sub-rectangulares, proyectadas fuertemente hacia adelante; distancia interaxilar usualmente mayor que la longitud de una axila; cada axila con una seta, raramente dos. Escutelo usualmente en forma de lentes biconvexos, más de 1.5 veces tan ancho como largo y usualmente con 4 setas, raramente con 6 o más setas. Parasitoides de escamas armadas (Diaspididae) y mosquitas blancas (Aleyrodidae). En México, esta tribu incluye los géneros Dirphys, Encarsia y Pteroptrix; la tribu es rica en especies de Encarsia.

\section{Género Dirphys Howard}

Cuenta con sólo 6 especies, se distribuye en la región Neotropical. En México se conocen 2 especies, las cuales también se hallan en Ecuador (D. encantadora Polaszek \& Hayat), Costa Rica o Trinidad (D. mexicanus Howard). Ambas especies son parasitoides de la mosquita blanca Ceraleurodicus altissimus (Quaintance) (Polaszek \& Hayat 1992).

\section{Género Encarsia Förster}

Encarsia Förster, 1878: 65-66.

Aspidiotiphagus Howard, 1894: 229.

Prospalta Howard, 1894b: 6.

Prospaltella Ashmead, 1904b: 126.

Mimatomus Cockerell, 1911: 464.

Doloresia Mercet, 1912b: 294-296.

Prospaltoides Brèthes, 1914: 12.

Aspidiotiphagus (Paraspidiotiphagus) Alam, 1956: 359.

Aleurodiphilus DeBach \& Rose, 1981: 659.

Encarsiella Hayat, 1983: 85.

Encarsia es el género megadiverso en Aphelinidae, contiene más de 430 especies en la fauna mundial (Noyes 2014). Sus especies son endoparasitoides de escamas armadas (fam. Diaspididae) y de mosquitas blancas (fam. Aleyrodidae). En México actualmente se conocen 108 especies, incluyendo 67 especies descritas entre $2001 \mathrm{y}$ 2014 (Myartseva et al. 2012, 2014).

\section{Género Pteroptrix Westwood}

Pteroptrix Westwood, 1833b: 344.
[Pterothrix Nees, 1834: 409. Corrección inválida]. [Gyrolasia Foerster, 1856: 145. Nombre de remplazo para Pterothrix Nees, pre-ocupado por Pterothrix de Candolle, en plantas]

Archenomus Howard, 1898: 136.

Casca Howard, 1907: 83.

Artas Howard, 1907: 85.

Hispaniella Mercet, 1911: 511.

Pteroptrichoides Fullaway, 1913: 27.

Apteroptrix Girault, 1915: 65.

Pseudopteroptrix Fullaway, 1918: 464.

Oa Girault, 1929: [4].

Aphelosoma Nikolskaya, 1963: 186.

Archenomiscus Nikolskaya, en Nikolskaya \& Yasnosh, 1966: 249.

Contiene 42 especies, está ampliamente distribuido ya que ocurre en África, Australia y en las regiones Paleártica, Oriental, Neártica y Neotropical (Noyes 2014). Alrededor de 20 especies de Diaspididae son hospederas de Pteroptrix. En México actualmente se conocen 3 especies, incluyendo 2 descritas del país (Myartseva, 2009) - $P$. gonzalezi Myartseva de Chiapas y P. mexicana Myartseva de Baja California Sur y Michoacán. Pteroptrix smithi (Compere) fue introducida al Estado de Morelos para el control biológico de la escama roja de Florida Chrysomphalus aonidum (L.), una plaga dañina a la citricultura (Myartseva et al. 2012).

\section{Tribu Physcini Yasnosh}

Diagnosis. Pronoto entero. Axilas muy pequeñas, triangulares, ampliamente separadas y no proyectadas hacia adelante o muy poco; cada axila con una seta. Lóbulos laterales del mesoescudo pequeños, en forma de trapecio. Mesopleura entera, es una placa grande sin divisiones. Antena con 7 segmentos. Vena estigmal delgada con sensilas en un arreglo específico -una sensila anterior pequeña y detrás de ella, 3 sensilas en línea. Parasitoides de escamas armadas y de escamas suaves.

\section{Género Mexidalgus Myartseva}

Este género fue descrito en 2014 como el tercer género de la tribu Physcini. La especie tipo, M. toumeyellus Myartseva, fue obtenida de la escama suave Toumeyella martinezi Kondo \& González en el cacto columnar Myrtillocactus geometrizans en el Estado de Hidalgo, México (Myartseva et al. 2014, Kondo \& Gonzalez 2014). Recientemente se ha colectado en México una segunda especie, M. veracruzensis (Myartseva et al., 2014). Este género comparte características de Coccophagus (tribu Coccophagini) y de Coccobius (tribu Physcini), por lo que su acomodo en Physcini es provisional. 


\section{Género Coccobius Ratzeburg}

Coccobius Ratzeburg, 1852: 195.

Physcus Howard, 1895a: 43.

Encyrtophyscus Blanchard, en De Santis, 1948: 192.

Physculus Yasnosh, 1977: 1115.

La fauna de este género de importancia económica fue estudiada taxonómicamente en África (Prinsloo 1995), India (Hayat 1998, Hayat \& Khan 2010) y China (Wang et al. 2013); Hayat (1984) estudió y redescribió algunas especies descritas por Girault y Howard, elaborando la primera clave para 48 especies. Actualmente, Coccobius consiste de 94 especies (Noyes 2014) y tiene distribución mundial. Los hospederos de Coccobius son escamas armadas (fam. Diaspididae). En México ocurren 3 especies, incluyendo a C. juliae Myartseva descrita del Estado de Tamaulipas (Myartseva, 2000). C. donatellae Pedata \& Evans controló con éxito a la escama de la palma Comstockiella sabalis (Comstock) en Sabal mexicana en Ciudad Victoria, Tamaulipas (Gaona-Garcia et al. 2001). La identificación de $C$. stanfordi (Howard) colectada en México necesita confirmación. En el Entomological Research Museum de la Universidad de California-Riverside, EU, se encuentran especímenes de Coccobius colectados hace muchos años en el Estado de Baja California Sur, México, los cuales requieren ser estudiados para conocer su identificación. En este artículo se describe una nueva especie de Coccobius de dicha entidad federativa.

\section{Descripción de la nueva especie Coccobius frontalis sp. nov. Myartseva}

Material. Holotipo, hembra: México, Baja California Sur, Los Cabos, Las Barracas, pan trap, 17.IV.1986 (col. P. DeBach), UCRC Ent. 54229. Holotipo depositado en UCRC, Riverside, California, EU.

Diagnosis. Coccobius frontalis sp. nov. difiere de las especies cercanas por la siguiente combinación de caracteres: escapo amarillo blancuzco con la parte basal ahumada, frontovértice blanco amarillento, pedicelo más largo que el primer segmento funicular, Ovipositor dos veces tan largo como la tibia media.

Hembra. Longitud del cuerpo: $0.6 \mathrm{~mm}$.

Coloración. Cara amarilla, frontovértice blanco amarilento, occipucio parduzco debajo del foramen, mandíbula parda. Antenas con radícula ahumada, escapo, pedicelo y segmentos flagelares 2, 3 y 5 amarillo blancuzco, segmento 1 negro, segmento 4 pardo. Lóbulo medio del mesoescudo blanco amarilento medialmente, parduzco en los lados. Escutelo parduzco, blanco amarilento basal y lateralmente. Pronoto pardo oscuro. Axilas y lóbulos late- rales parduzcos. Mesopleura pardo oscuro. Alas anteriores hialinas. Patas parduzcas, ápice de las coxas medias blancuzcos, 1/3 apical de los fémures anteriores y medios $\mathrm{y}$ todas las tibias blancas, tarsos blancos, segmento apical ligeramente ahumado. Terguitos gástricos 1-3 amarillo blancuzcos medialmente, los demás terguitos parduzcos, más oscuros en los lados. Ovipositor pardo, segundo valvífer amarillo blancuzco.

Estructura. Cabeza ligeramente más ancha que el mesosoma, 1.2 veces tan ancha como alta. Frontovértice cerca de 0.6 veces tan ancha como la anchura de la cabeza. Ocelos en triángulo obtuso. Distancia del ocelo posterior al ojo cerca de 0.4 veces tan larga como la distancia entre los ocelos posteriores. Mandíbula con dos dientes y una parte truncada corta. Palpos labiales y maxilares inusualmente cortos. Ojos ligeramente más cortos que las mejillas. Antenas (Fig. 1) insertadas debajo del nivel del margen inferior de los ojos. Distancia entre tórulos similar a la distancia del tórulo al margen bucal y dos veces tan larga como la distancia al ojo. Radícula antenal 2.6 veces tan larga como ancha. Escapo cerca de 4 veces tan largo como ancho. Pedicelo 1.7 veces tan largo como ancho. Segmentos funiculares 1 y 2 subiguales en longitud y en anchura, alrededor de 1.4 veces tan largos como anchos y más cortos que el pedicelo (11:14); segmento 3 ligeramente más largo y cerca de 1.5 veces tan largo como ancho. Segmentos de la maza antenal muy ligeramente más anchos y más largos que el funículo, su segmento apical cerca de 1.7 veces tan largo como el segmento basal. Segmentos funiculares con una sensila longitudinal cada uno, segmentos de la maza antenal con una sensila cada uno. Lóbulo medio del mesoescudo con muchas setas cortas y delgadas; axila con una seta, lóbulo lateral con dos setas delgadas. Escultura del lóbulo medio con celdas fuertemente elongadas medialmente, reticuladas en la base y en los lados; escultura del escutelo también con celdas fuertemente elongadas medialmente, reticuladas en la base y no es clara en los lados. Ala anterior alrededor de 2.8 veces tan larga como ancha, su fleco marginal cerca de 0.2 veces tan largo como la anchura máxima del ala. Vena marginal ligeramente más corta que la vena submarginal, con 9 setas a lo largo del margen anterior; vena submarginal con 6 setas delgadas más largas. Fórmula tarsal 5-5-5. Espuela de la tibia media más o menos tan larga como el segmento funicular 3. Ovipositor expuesto, dos veces tan largo como la tibia media (Figs 2, 3); válvula 3 cerca de 0.5 veces tan larga como el valvífer 2 y cerca de 2.5 veces tan larga como el basitarso de la pata media. 

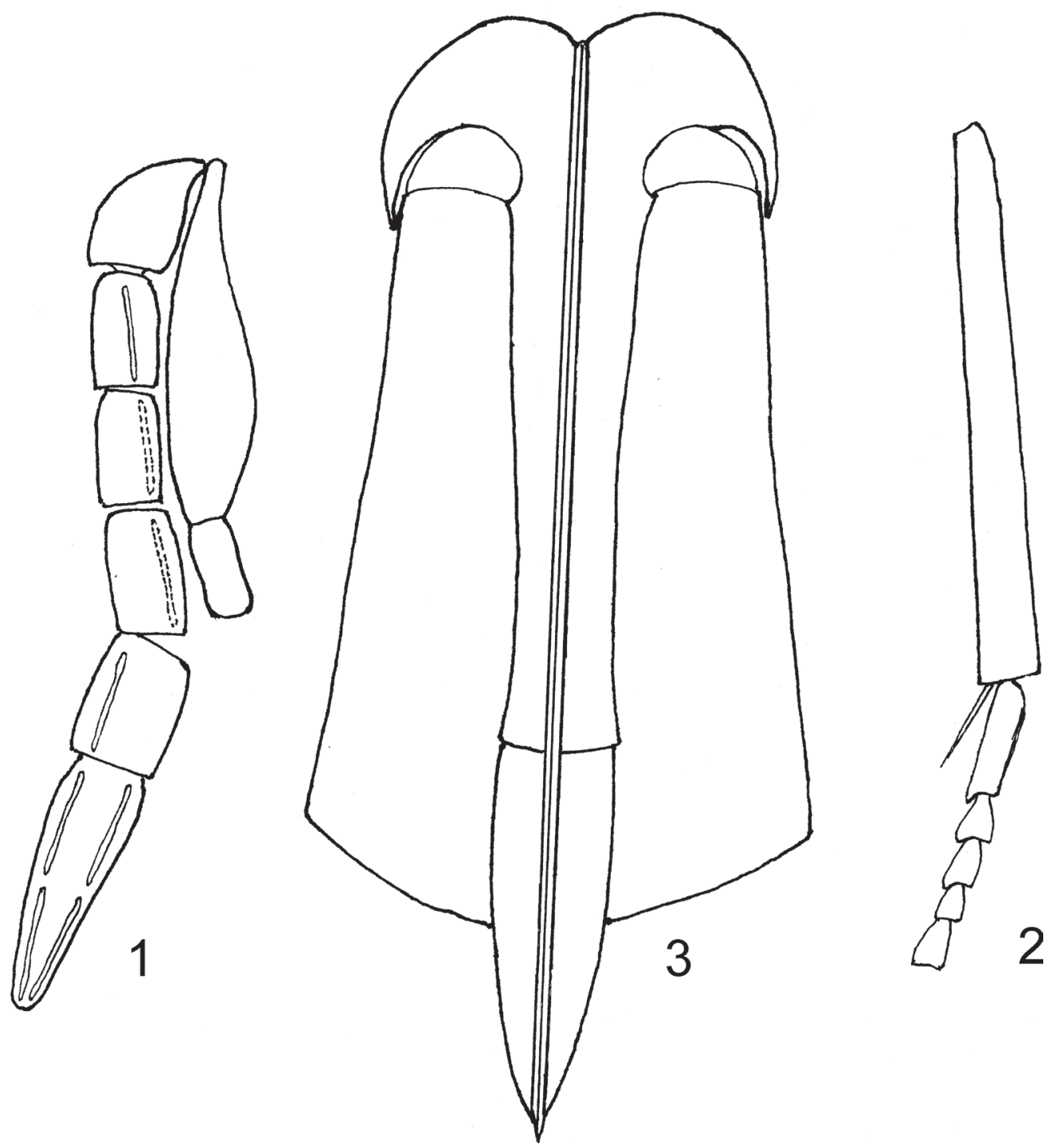

Figuras 1-3. Coccobius frontalis sp. nov., hembra: 1-antena, 2-tibia media y tarso, 3-ovipositor.

\section{Macho desconocido}

Comentarios. Coccobius frontalis sp. nov. es cercana a la especie neártica C. stanfordi (Howard) y a la especie oriental C. reticulatus (Compere \& Annecke). De C. stanfordi difiere en lo siguiente: en $C$. frontalis, el escapo es amarillo blancuzco con la parte basal ahumada, frontovértice y valvífer 2 blanco amarillento; pedicelo visiblemente más largo que el segmento funicular 1, el cual es más corto que el segmento 3; maza antenal más larga que el funículo; válvula 3 es 2.5 veces tan larga como el basitarso medio. En C. stanfordi, el escapo, frontovértice y el valvífer 2 son pardo oscuro; pedicelo más corto segmento funicular 1, el cual es más largo que el segmento; maza antenal más corta que el funículo; válvula 3 es 1.7 veces tan larga como el basitarso medio.

Las diferencias entre $C$. frontalis sp. nov. y $C$. reticulatus son: en $C$. frontalis, el frontovértice es 0.6 veces tan ancho como la anchura de la cabeza; segmento funicular 1 más corto que el pedicelo; ovipositor dos veces tan largo como la tibia media; valvífer 2 es 2.1 veces tan largo como la válvula 3 mientras que en $C$. reticulatus, el frontovértice es 0.5 veces tan ancho como la anchura de la cabeza; segmento funicular 1 igual o más largo que el pedicelo; ovipositor 1.6-1.8 veces tan largo como la tibia media; valvífer 2 es $2.8-3.0$ veces tan largo como la válvula 3 . 
AGRADECIMIENTOS. Al Dr. S. V. Triapitsyn (Entomological Research Museum, Universidad de California-Riverside, EU), por el préstamo de material mexicano de Coccobius. A los revisores de este artículo, por sus atinadas sugerencias. A la Facultad de Ingeniería y Ciencias, UAT, por su apoyo para efectuar esta investigación.

\section{LITERATURA CITADA}

Gaona-García, G., Myartseva, S. N. \& Ruiz-Cancino, E. 2001. Enemigos naturales de la escama de la palma Comstockiella sabalis (Homoptera: Diaspididae) en Tamaulipas, México. Memorias XXIV Congreso Nacional de Control Biológico, México, pp. 121122.

Hayat, M. 1984. Notes on some species of Coccobius and Prophyscus (Hymenoptera: Aphelinidae), with special reference to Girault and Howard types. Oriental Insects, 18: 289-334.

Hayat, M. 1998. Aphelinidae of India (Hymenoptera: Chalcidoidea): a taxonomic revision. Memoirs on Entomology, International. Associated Publishers, Gainesville, Florida, USA, 13: 1-416.

Hayat, M. \& Khan, F. R. 2010. Additions to the Aphelinidae of India (Hymenoptera -Chalcidoidea): 1. On species of Ablerus Howard, Coccobius Ratzeburg, Coccophagus Westwood, Pteroptrix Westwood and Idiococcobius Hayat gen. nov. Colemania, 21: 1-31.

Kondo, T. \& Gonzalez, H. 2014. A new species of Toumeyella Cockerell (Hemiptera: Coccidae) on Myrtillocactus geometrizans (Cactaceae) from Mexico with a checklist of known species of Toumeyella in the world. Insecta Mundi, 03961-10.

Myartseva, S. N. 2000. The genus Coccobius new for Mexico, with description of a new species and key to Nearctic species (Hymenoptera: Aphelinidae). Zoosystematica Rossica, 8: 331-334.
Myartseva, S. N. 2009. Two new species of Pteroptrix Westwood, 1833 (Hymenoptera: Aphelinidae) from Mexico. Zoosystematica Rossica, 18: 102-108.

Myartseva, S. N., Ruiz-Cancino, E. \& Coronado-Blanco, J. M. 2012. Aphelinidae (Hymenoptera: Chalcidoidea) de importancia agrícola en México. Revisión y claves. Serie Avispas Parasíticas de Plagas y otros Insectos No. 8. Universidad Autónoma de Tamaulipas. México. 413 pp. Publicación en CD.

Myartseva, S. N., Coronado-Blanco, J. M., Lomeli-Flores, J. R. \& Martinez-Hernández, D. Y. 2014. A new genus for a new species of the family Aphelinidae (Hymenoptera: Chalcidoidea) from Mexico. Zoosystematica Rossica, 23: 131-136.

Myartseva, S. N., García-Guerrero, D. A., Ruiz-Cancino, E. \& Coronado-Blanco, J. M. 2014. A new parasitoid species (Hymenoptera: Chalcidoidea: Aphelinidae) of a soft scale insect (Hemiptera: Coccidae) from Mexico. Zoosystematica Rossica, 23: 264-268.

Noyes, J. S. 2014. Universal Chalcidoidea Database [online]. Worldwide Web electronic publication. www.nhm.ac.uk/entomology/ chalcidoids/index.html (last updated: August 2014).

Polaszek, A. \& Hayat, M. 1992. A revision of the genera Dirphys Howard and Encarsiella Hayat (Hymenoptera: Aphelinidae). Systematic Entomology, 17: 181-197.

Prinsloo, G. L. 1995. Revision of the Southern African species of Coccobius Ratzeburg (Hymenoptera: Aphelinidae), parasitoids of armored scale insects (Homoptera: Diaspididae). Journal of Natural History, 29: 1517-1541.

Wang, Z. H., Huang, J. \& Pan, D. M. 2013. Taxonomic review of Coccobius species (Hymenoptera: Aphelinidae) from China, with notes on their use in biological control of scale insect pests (Hemiptera: Diaspididae), and description of a new species. Journal of Natural History, 48: 359-373. 